\title{
X-ray study of the liquid potassium surface: Structure and capillary wave excitations
}

\author{
Oleg Shpyrko, Patrick Huber, Alexei Grigoriev, and Peter Pershan \\ Department of Physics, Harvard University, Cambridge, Massachusetts 02138 \\ Ben Ocko \\ Department of Physics, Brookhaven National Lab, Upton, New York 11973 \\ Holger Tostmann \\ Department of Materials Science and Engineering, University of Florida, Gainsville, Florida 32611 \\ Moshe Deutsch \\ Department of Physics, Bar-Ilan University, Ramat-Gan 52900, Israel
}

(Received 26 August 2002; published 14 March 2003)

\begin{abstract}
We present X-ray reflectivity and diffuse scattering measurements from the liquid surface of pure potassium. They strongly suggest the existence of atomic layering at the free surface of a pure liquid metal with low surface tension. Prior to this study, layering was observed only for metals like $\mathrm{Ga}$, In, and $\mathrm{Hg}$, the surface tensions of which are five- to sevenfold higher than that of potassium, and hence closer to inducing an ideal "hard wall" boundary condition. The experimental result requires quantitative analysis of the contribution to the surface scattering from thermally excited capillary waves. Our measurements confirm the predicted form for the differential cross section for diffuse scattering, $d \sigma / d \Omega \sim 1 / q_{x y}^{2-\eta}$ where $\eta=k_{B} T q_{z}^{2} / 2 \pi \gamma$, over a range of $\eta$ and $q_{x y}$ that is larger than any previous measurement. The partial measure of the surface structure factor that we obtained agrees with computer simulations and theoretical predictions.
\end{abstract}

DOI: 10.1103/PhysRevB.67.115405

PACS number(s): 61.25.Mv, 61.10.-i

\section{INTRODUCTION}

In contrast to measurements of dielectric liquids, which show a monotonically varying density profile at the liquidvapor interface, liquid metals exhibit a phenomenon known as surface-induced layering. ${ }^{1,2}$ This had been thought to occur because the Coulomb interactions between two constituents of the liquid metal, the free-electron Fermi gas $^{3}$ and the classical gas of positively charged ions, suppress local surface fluctuations that would otherwise conceal the layered atomic ordering that occurs at hard walls. ${ }^{4}$ On the other hand, recent molecular simulations by Velasco et al. ${ }^{5}$ argue that surface layering should occur in any liquid for which the ratio of melting temperature $T_{m}$ and critical temperature $T_{c}$, $T_{m} / T_{c}$, is sufficiently small. Surface-induced layering for liquid metals was theoretically predicted by Rice et al. ${ }^{1}$ later confirmed by density-functional calculations ${ }^{2,4}$ and computer simulations, ${ }^{6}$ and more recently observed experimentally in a number of pure liquid metallic systems such as $\mathrm{Hg},{ }^{7} \mathrm{Ga},{ }^{8} \mathrm{In},{ }^{9}$ as well as in binary alloys. ${ }^{10,11}$ In fact, for $\mathrm{Ga}, \mathrm{Hg}$, and $\mathrm{K}$ $T_{m} / T_{c} \approx 0.13-0.15$ and these observations are consistent with Velasco et al. ${ }^{5}$ From another point of view, all but one of the metallic systems studied so far exhibit relatively high values of surface tension, a few hundred $\mathrm{mN} / \mathrm{m}$, leaving unanswered the fundamental question of whether the surfaceinduced layering is caused by the high surface tension, which suppresses the long-wavelength capillary waves, or whether it is caused by the intrinsic metallic properties of the studied systems. In the latter case surface-induced layering should also be found in low-surface tension metals. Monovalent alkali metals have a surface tension comparable to that of water $(72 \mathrm{mN} / \mathrm{m})$ and their study should therefore shed light on this issue. Moreover, monovalent alkali metals are the best available examples of an ideal nearly free-electron metal, ${ }^{12}$ therefore they are particularly well suited for studying surface induced layering in an ideal, simple liquid metal. The same properties which make alkali metals ideal for studies of layering have also been the motivation for the numerous computer simulations of liquid $\mathrm{Na}, \mathrm{K}$, and Cs. ${ }^{6,12-16}$

Until recently, experimental surface studies of the pure liquid alkalis were thought to be impossible because of two fundamental experimental problems. First, their high evaporation rates which lead to high values of vapor pressure, ${ }^{17}$ and high reactivity ${ }^{18}$ to water and oxygen implied that their surfaces could not be maintained atomically clean for extended periods of time even under UHV conditions. Therefore the first study of alkali-metal systems performed by our group $^{19}$ employed a binary $\mathrm{KNa}$ alloy that preserves most of the characteristic features typical of a nearly free-electron metal, but for which the vapor pressure and melting temperature were both lower than those of the pure metals. Contrary to expectations, no measurable changes were detected in that study in the x-ray reflectivity of the alloy's surface over periods of many hours or after intentional exposure to large doses of oxygen. This lack of surface contamination is believed to be due to the high solubility of the oxides of these metals in the bulk liquid, ${ }^{18,20}$ and to the low surface tension of liquid alkali metals which does not promote segregation of the oxide at the surface. The fact that the surface of the $\mathrm{KNa}$ alloy was found to remain atomically clean for extended periods of time suggests that the same might be true for the pure metals. The second experimental problem is that the large capillary-wave-induced roughness due to the low surface tension renders the $\mathrm{x}$-ray reflectivity peak weak and 


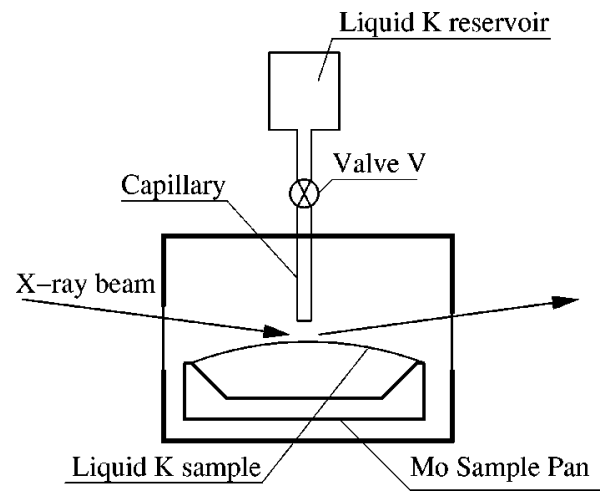

FIG. 1. Schematic description of the experimental setup.

inseparable from its diffuse wings. Thus a clear layering peak in the measured reflectivity curve, such as found in the high-surface-tension liquid metal studies, ${ }^{7,8,10,11,19}$ cannot be observed here. We demonstrate here that this problem can be resolved by a subtle application of diffuse scattering and reflectivity measurements. A significant aspect of this paper pertains therefore to the methods of measurement and analysis that are necessary in order to separate the effects due to the thermal capillary waves from those of the surfaceinduced layering. Using these methods, the present study clearly demonstrates the existence of surface induced layering in liquid $\mathrm{K}$, with properties similar to those of the highsurface-tension liquid metals studied previously. .,8,10,11,19 $^{2}$

\section{EXPERIMENTAL DETAILS}

The sample preparation procedure and the experimental setup were similar to our previous experiment on the $\mathrm{KNa}$ alloy. ${ }^{19}$ The sample was prepared in an argon-filled glove box $\left(<2 \mathrm{ppm} \mathrm{H}_{2} \mathrm{O},<1 \mathrm{ppm}_{2}\right)$. The $\mathrm{K}$ with purity of $99.999 \%$, which was contained in a glass ampoule, was melted and then transferred into a sealed stainless-steel reservoir inside the glove box using a glass syringe. The reservoir was sealed with a Teflon o ring and then mounted onto a UHV chamber. After a 24-h bakeout of the entire chamber to $150^{\circ} \mathrm{C}$, a base pressure of $10^{-9}$ Torr has been established inside the UHV chamber. The $\mathrm{K}$ in the reservoir was then heated above the melting temperature $T_{m}=63{ }^{\circ} \mathrm{C}$ and dropped through a capillary into a Mo sample pan mounted inside the UHV chamber by opening the valve $\mathrm{V}$ shown on Fig. 1. Previous to filling the pan with liquid K, the pan was cleaned by sputtering with $\mathrm{Ar}^{+}$ions for several hours in order to remove the native Mo oxide. This latter procedure significantly improves the wetting of the sample pan by liquid $\mathrm{K}$ and therefore helps to achieve a larger area of macroscopically flat liquid surface than it has been possible for high surface tension metals. ${ }^{8}$ The sample pan was kept at a constant temperature of $70^{\circ} \mathrm{C}$. Pure $\mathrm{K}$ has a vapor pressure of about $10^{-6}$ Torr at this temperature, which renders the requested UHV conditions almost impossible. We circumvented this problem by a differential pumping setup, where we used a permanently attached ion pump. By constantly pumping at the sample chamber, we were able to achieve a base pressure of about $10^{-9}$ Torr in the chamber, three or-

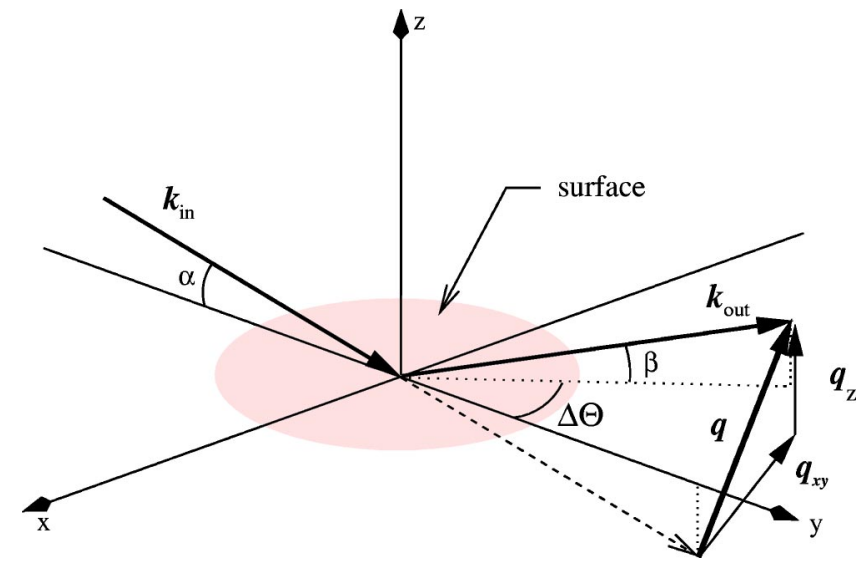

FIG. 2. Kinematics of the x-ray measurement.

ders of magnitude lower than vapor pressure of $\mathrm{K}$.

Measurements were carried out using the liquid surface reflectometer at CMC-CAT beam-line at the Advanced Photon Source, Argonne National Laboratory, operating at an $\mathrm{x}$-ray wavelength of $1.531 \AA^{-1}$. The kinematics of the measurement is illustrated in Fig. 2. Two different surfacesensitive $\mathrm{x}$-ray techniques were applied-specular x-ray reflectivity and x-ray diffuse scattering. In the specular x-ray reflectivity geometry, the incident angle $\alpha$ and the reflection angle $\beta=\alpha$ are varied simultaneously while keeping $\Delta \Theta$ $=0$ constant. The signal is measured as a function of the surface-normal momentum transfer $q_{z}=2 \pi / \lambda(\sin \alpha+\sin \beta)$ with the in-plane momentum transfer $q_{x y}=0$.

In the $\mathrm{x}$-ray diffuse scattering geometry, the detection angle $\beta$ is varied within the plane of incidence $\Delta \Theta=0$, while the incident angle $\alpha$ stays fixed. The observed scattering therefore follows a trajectory in the $\left(q_{x}, q_{z}\right)$ plane with $d q_{z} / d q_{x}=\tan \beta$ and $q_{y}=0$. The signals measured in the two geometries are, however, related since the signal measured at $\beta=\alpha$ in the diffuse measurement is the specular signal.

The differential cross section of the diffuse scattering signal is given $b y^{21}$

$$
\frac{d \sigma}{d \Omega}=\frac{A_{0}}{\sin \alpha}\left(\frac{q_{c}}{2}\right)^{4} \frac{k_{B} T}{16 \pi^{2} \gamma}\left|\Phi\left(q_{z}\right)\right|^{2} \frac{1}{q_{x y}^{2-\eta}}\left(\frac{1}{q_{\max }}\right)^{\eta},
$$

where $q_{c}$ (Ref. 22) is the critical angle for total external reflection of $\mathrm{x}$ rays, $\gamma$ is the surface tension, $q_{\max } \approx \pi / \xi$ is the value of the upper cutoff for capillary wave contributions. $^{21,23}$ The value for $\xi$ is empirically taken to be the nearest-neighbor atomic distance in the bulk. ${ }^{22,24}$ The scattering line-shape exponent $\eta$ is given by ${ }^{25}$

$$
\eta=\frac{k_{B} T}{2 \pi \gamma} q_{z}^{2}
$$

The principal goal of the present surface $\mathrm{x}$-ray scattering measurements is to determine the surface structure factor ${ }^{8}$

$$
\Phi\left(q_{z}\right)=\frac{1}{\rho_{\infty}} \int d z \frac{\langle d \rho(z)\rangle}{d z} \exp \left(\iota q_{z} z\right)
$$


which is given by the Fourier transform of the $(x, y)$-averaged intrinsic density profile $\langle\rho(z)\rangle$ along the surface normal $z$ in the absence of capillary waves. ${ }^{26}$ In this equation, $\rho_{\infty}$ is the bulk electron density. The fact that the peak of the differential cross section at the specular condition is algebraic, rather than a $\delta$ function, complicates the determination of $\Phi\left(q_{z}\right)$ from the measured intensity. This can be done, however, when both specular reflectivity and diffuse scattering measurements are available.

\section{PRACTICAL CONSIDERATIONS}

It follows from Eq. (1) that the nominal specular reflectivity signal, observed at $\alpha=\beta$ and $\Delta \Theta=0$, corresponds to the integral of the $\left(1 / q_{x y}\right)^{2-\eta}$ over the area $\Delta q_{x y}^{\text {res }}\left(q_{z}\right)$ $=\Delta q_{x}^{\text {res }} \times \Delta q_{y}^{\text {res }}$ which is the projection of the instrumental resolution function on the horizontal plane of the liquid surface. The $q_{z}$ dependence of $\Delta q_{x y}^{\text {res }}\left(q_{z}\right)$ is due to the increase of the projected width of the detector resolution $\left|\Delta q_{y}^{\text {res }}\right|$ $=(2 \pi / \lambda) \sin \beta \Delta \beta$ on the plane of the surface as $\beta$ increases. The integration results in the reflectivity scaling as $\left(\Delta q_{y}^{\text {res }} / q_{\text {max }}\right)^{\eta} / \eta$ and since $\eta$ scales as $q_{z}^{2}$, the net effect is a Debye-Waller-like decrease in the reflectivity as $q_{z}$ increases. For example, if one defines a mean-square surface roughness by taking an average over a length scale $\approx\left(\Delta q_{y}^{\text {res }}\right)^{-1}$, this roughness can be written as ${ }^{27}$

$$
\sigma^{2}\left(q_{z}\right)=\left(k_{b} T / 2 \pi \gamma\right) \ln \left(q_{\max } / \Delta q_{y}^{\mathrm{res}}\right) .
$$

From this one can show that the reflectivity is proportional to a Debye-Waller factor $\exp \left[-q_{z}^{2} \sigma^{2}\left(q_{z}\right)\right]$. The important point to note is that for liquid surfaces the signal measured at the specular condition $q_{x y}=0$ depends on the resolution of the reflectometer.

The validity of the simple capillary wave model in the high $q$ range remains controversial and untested. Although the model assumes that the energy for surface excitations can be described by the capillary form $\approx \gamma / q_{x y}^{2}$ with a constant surface tension $\gamma$, over the entire range of length scales from the macroscopic to a microscopic length of the order of $\xi$ $=\pi / q_{\max }$, where $\xi$ is normally taken to be of the order of the interatomic/molecular spacing $a$, this is certainly not strictly true for lengths that are shorter than a length that we might designate as $\xi_{\text {cutoff }}=\pi / q_{\text {cutoff }}>a .^{28}$ Nevertheless, the basic physics is not changed if we replace $q_{\max }$ by the smaller $q_{\text {cutoff }}$ since the differences engineered by this are really just a matter of accounting, in the sense that it determines the relative contributions between the capillary wave and structure factor terms given in Eq. (1). Nevertheless, this is not a serious objection to the present treatment, since the capillary wave model is certainly accurate for values of $q_{x y}$ covered by the present measurements. Even if the model were to fail for values of $q_{x y}>q_{\text {cutoff }}$, the shorter wavelength fluctuations could be incorporated into the definition of a $q_{\text {cutoff }}$-dependent structure factor $\Phi\left(q_{z}\right)$. On the other hand, the fact that the intensity of off-specular diffuse scattering predicted by Eq. (1) has been shown to be in satisfactory quantitative agreement with measurements for a number of liquids over a wide range of $q_{y}$ and $q_{z}$ implies that $q_{\text {cutoff }}$ is reasonably close to $q_{\max }=\pi / a$ and the extracted structure factor $\Phi\left(q_{z}\right)$ is a reasonable measure of the local profile.

There are three practical considerations involved in these measurements. The first is that it is necessary to insure that the measurement corresponding to the integral over the resolution does not include a diffuse scattering contribution from sources other than the surface. For flat surfaces, for which the specular reflectivity can be described by a $\delta$ function at $q_{x y}=0$, this can be accomplished by subtracting the scattering measured at some finite value of $q_{x y}$ from the signal measured at $q_{x y}=0$. For liquid surfaces this separation is complicated by the fact that the signal measured at finite $q_{x y}$ includes scattering from the $1 / q_{x y}^{2-\eta}$ tails of the cross section. We circumvent this problem in the present study by comparing the difference between measurements at $q_{x y}=0$ and at some $q_{x y} \neq 0$ with the same difference as predicted by the theoretical differential cross section [Eq. (1)]. Thus we present all data in this study as differences between a signal measured at $\{\alpha, \beta, 0\}$ and at $\left\{\alpha, \beta, \Delta \Theta_{\text {offset }}=0.2^{\circ}\right\}$. For specular reflectivity $\alpha=\beta$; however, for off-specular diffuse measurements $\beta \neq \alpha$. Diffuse scattering from all sources other than the surface are essentially constant over this range of $\Delta \Theta_{\text {offset }}$. This is because these other sources of scattering, such as diffuse scattering from either the bulk liquid or from the vapor above the surface, only depend on the absolute value of the total momentum transfer $|q|$ and this is essentially unchanged for small offsets in $\Delta \Theta_{\text {offset }}$.

The second practical consequence of the $1 / q_{x y}^{2-\eta}$ form is that as $\eta$ increases it becomes increasingly difficult to distinguish the singular specular peak from the off-specular power-law wings. At $\eta \geqslant 2$ this distinction is no longer possible, ${ }^{21}$ even with infinitely sharp resolution. Unfortunately, for liquid $\mathrm{K}, \eta$ reaches the limit of 2 at $q_{z}$ $=1.74 \AA^{-1}$, which is comparable to $q_{z}=1.6 \AA^{-1}$, where the quasi-Bragg surface layering peak is expected to be observed. However, in the following paragraph we show that as a result of resolution effects, the limit at which the specular cusp can no longer be distinguished from the background in practice is even lower.

The impact of the finite resolution can be easily seen by considering a simple example of a rectangular resolution function of infinite length along the $x$ axis but with a very small width along the $y$ axis. For small values of $\alpha$ this is often an excellent approximation to the real resolution functions. ${ }^{29}$ With this approximation, the integration over the $q_{x}$ component yields a $1 / q_{y}^{1-\eta}$ dependence for the diffuse scattering, placing the value of $\eta$ where the specular ridge disappears to be $\eta=1$ instead of $\eta=2$. For $\mathrm{K}$, this $\eta=1$ limit is reached at $q_{z}=1.2 \AA^{-1}$. The detector slit dimensions which provide an optimal compromise between intensity and resolution were discussed by one of $\mathrm{us}^{21}$ and are (width) $\approx$ (height) $\times \sin \alpha$, where $\alpha$ is the incident angle.

The third practical problem in extending x-ray reflectivity measurements to large $q_{z}$ is the fast falloff of the reflected intensity. Much more serious is the Debye-Waller-like factor that was discussed above. Since the surface tension $\gamma$ for K $\left(110 \mathrm{mN} / \mathrm{m}\right.$ at $\left.70{ }^{\circ} \mathrm{C}\right)$ is much lower than that of a metal like $\mathrm{Ga}(770 \mathrm{mN} / \mathrm{m}),{ }^{17}$ the factor of $\exp \left[-\sigma^{2} q_{z}^{2}\right]$ with $\sigma^{2} \sim 1 / \gamma$ is 


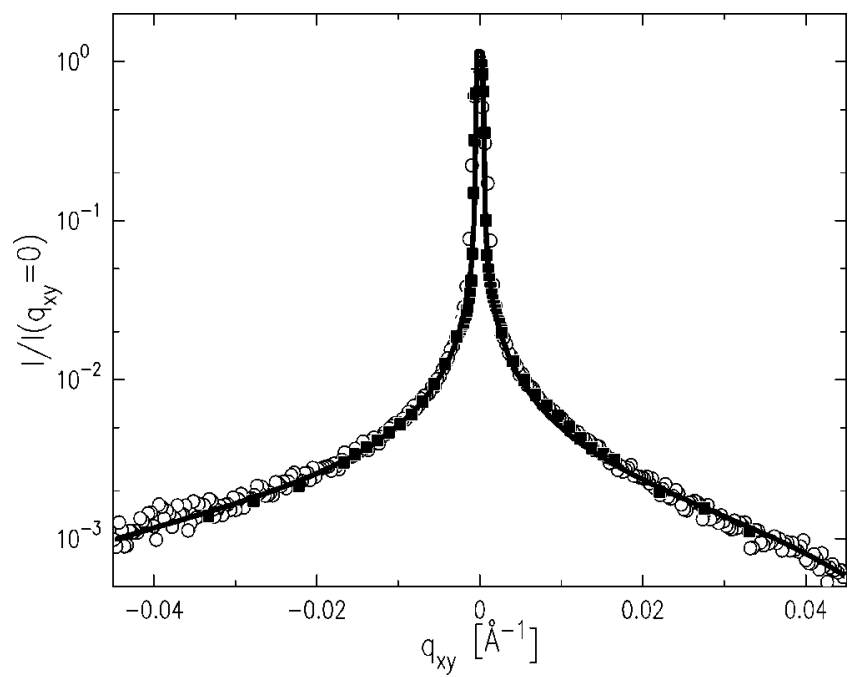

FIG. 3. Comparison of $\mathrm{x}$-ray diffuse scattering scans taken with point detector-filled squares, and position sensitive detector (PSD) - open circles. The specular condition corresponds to $q_{z}$ $=0.3 \AA^{-1}$. The resolution of the bicron detector $(0.22 \mathrm{~mm}$ vertically and $2.5 \mathrm{~mm}$ horizontally) was chosen to match that of a PSD. Also shown is a line which represents theoretical modeling using the capillary-wave theory for given resolution, temperature $\left(70{ }^{\circ} \mathrm{C}\right)$ and surface tension $(110 \mathrm{mN} / \mathrm{m})$.

orders of magnitude smaller than that of $\mathrm{Ga}$ at the same values of $q_{z}$. Even at high-flux synchrotron facilities such as the Advanced Photon Source the low count rates of the reflectivity signal observed at $q_{z} \approx 1.0-1.1 \AA^{-1}$ are among the dominant limiting factors of the $q_{z}$ range accessible in this experiment.

\section{MEASUREMENTS}

To partially compensate for the low intensity the offspecular diffuse scattering measurements were carried out using a linear position sensitive detector (PSD), rather than a point detector. In Fig. 3 we show a diffuse scattering profile as a function of $q_{x y}$. The specular condition corresponds to $q_{z}=0.3 \AA^{-1}$. The profile exhibits a sharp peak whose width is dominated by the instrumental resolution, however, the long tails are an intrinsic property of the capillary wave surface roughness. We note that the data shown represents the intensity difference for data obtained at $\Delta \Theta=0$ and $\Delta \Theta=$ $\pm \Delta \Theta_{\text {offset }}$ with either a position sensitive detector (PSD) or with a point detector. An advantage of the PSD configuration is that it permits the entire profile to be acquired simultaneously for a wide range of the output angles $\beta$ for a fixed $\alpha$. We carefully checked for possible instrumental errors associated with the PSD (saturation or nonlinear effects) by comparing data obtained with both the PSD and a point detector. As shown in Fig. 3, data obtained with both configurations are indistinguishable. Further, this comparison allowed an accurate determination of the PSD spatial resolution, about $0.22 \mathrm{~mm}$ vertically. To provide for a reasonable comparison with the point detector, the vertical slit was set to match the resolution of the PSD. The solid line in Fig. 3 represents the theoretical prediction for the different intensity, obtained by

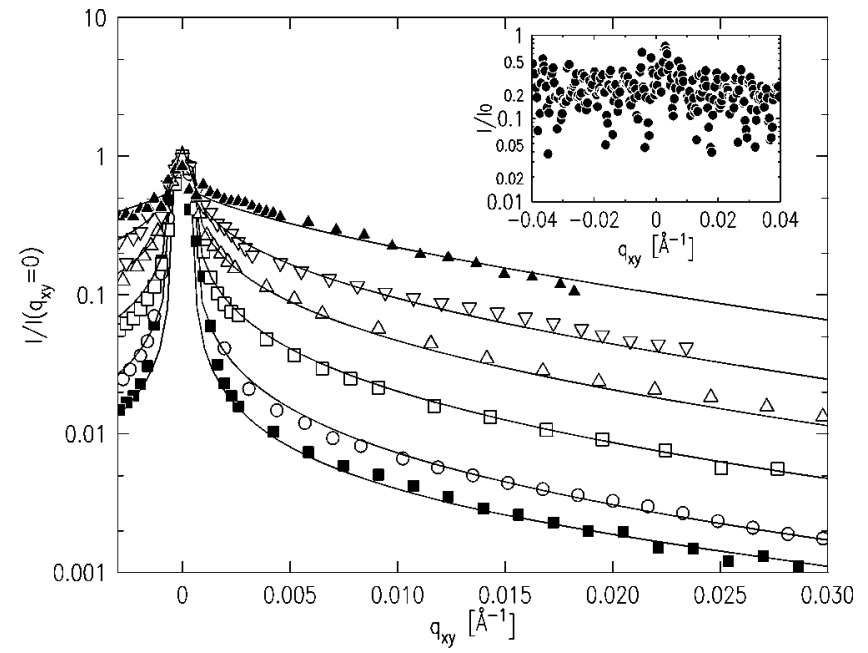

FIG. 4. X-ray diffuse scattering scans taken with PSD, as a function of wave-vector transfer component $q_{x y}$. Specular condition corresponds to values of $q_{z}$ (bottom to top): $0.3,0.4,0.6,0.8$, 1.0 , and $1.1 \AA^{-1}$. For comparison, the scans are normalized to unity at $q_{x y}=0$. Lines represent theoretically simulated scans for given values of $q_{z}$. The inset shows a similar scan taken at $1.2 \AA^{-1}$, for which the specular peak is marginally observable.

numerically integrating $d \sigma / d \Omega$ in Eq. (1) over the known resolution. The known values of the incident angle $\alpha$, temperature $T$, surface tension $\gamma, \mathrm{x}$-ray energy, and detector resolution were used without any adjustable parameters. The essentially perfect agreement between both experimental data sets and the theoretical simulations strongly supports the theoretical model for the effect of the thermally induced surface excitation on the diffuse $\mathrm{x}$-ray scattering from the surface.

Further diffuse scattering data were collected with the PSD, which proved to be especially useful at higher values of $q_{z}$, where the weaker scattering makes it increasingly more difficult to separate the specular signal from the powerlaw wings as $\eta$ approached 1 . Figure 4 shows a set of diffuse scattering scans measured with a PSD and the corresponding calculated theoretical predictions, for several $q_{z}$ values $(0.3$, $0.4,0.6,0.8,1.0$, and $\left.1.1 \AA^{-1}\right)$. Each curve represents a difference between a diffuse measurement in the plane of incidence $(\Delta \Theta=0)$ and an average of two measurements taken by displacing the detector out of the plane of incidence by an angle of $\Delta \Theta_{\text {offset }}= \pm 0.2^{\circ}$. Theoretical simulations include the same subtraction operation as well. All data sets are normalized to unity at $q_{x y}=0$ for easier comparison. As $q_{z}$ gets larger, the ratio of specular signal to the diffuse wings decreases, and the specular peak eventually disappears under the off-specular diffuse wings. In the curve measured at $\left(q_{z}\right.$ $\left.=1.2 \AA^{-} 1\right)$, shown in the inset of Fig. 4, the specular peak all but disappears. The solid lines that represent numerical integration of the capillary-wave theory [Eq. (1)] show a remarkably good agreement with experimental data. The only adjustable quantity in these theoretical curves is the form of $\Phi\left(q_{z}\right)$. As discussed below, one common form was used for all data sets.

This agreement provides additional support for the absence of surface contamination, since the presence of any 


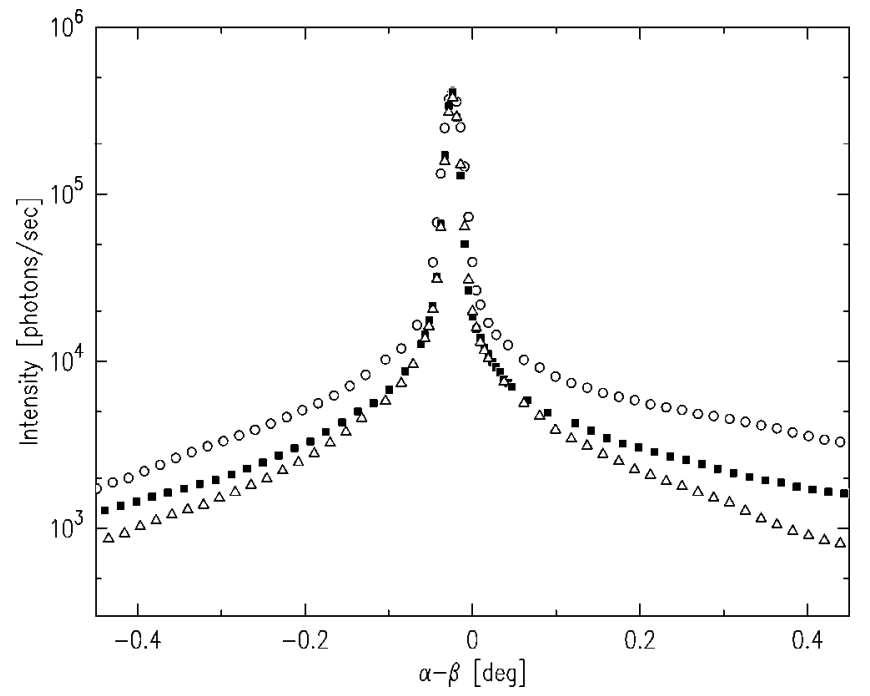

FIG. 5. Effects of varying the horizontal resolution function on the diffuse scattering data. Diffuse scans taken with PSD, peak position corresponding to $q_{z}=0.4 \AA^{-1}$, as a function of angular deviation from specular condition $(\alpha-\beta)$ with fixed vertical resolution $(0.22 \mathrm{~mm})$, while horizontal resolution was changed (bottom to top: $2,4,8 \mathrm{~mm}$ ).

kind of inhomogeneous film at the surface would manifest itself in extra x-ray diffuse signal which could not be explained by capillary wave theory alone. Figure 5 shows the effect of varying the shape of the detector resolution on the diffuse scattering data. Changing the horizontal size of the slit in front of the PSD has little effect on either the measured intensity of the specular peak, because of the intrinsic property of the singularity, or on the diffuse scattering at small $q_{x y}$, because it is sharply peaked around $q_{x y}=0$. However, far away from the specular condition, the signal is directly proportional to the area of the resolution function, and scales with the size of the slits.

The excellent agreement between the experimental measurements and the theoretical simulations strongly supports our conclusion that by the methods above one can account properly and accurately for both the capillary-wave-induced diffuse scattering in, and the resolution effects on, the measured data. We have therefore employed these results to analyze the specular reflectivity data, to find out whether surface-induced layering exists at the surface of K. Specular reflectivity measurements were made by scanning $\alpha=\beta$ at both $\Delta \Theta=0$ and $\Delta \Theta= \pm \Delta \Theta_{\text {offset }}$. The $\beta$ resolution, $\Delta \beta$ $=0.2^{\circ}$, was set by fixing the height of the detector slit at 2.5 $\mathrm{mm}$, located $714 \mathrm{~mm}$ away from the center of the sample. Likewise, the horizontal resolution was defined by a 2.5mm-wide slit located at the same position. The ratio of the difference between the specular signal measured at $\Delta \Theta=0$ and at $\Delta \Theta= \pm \Delta \Theta_{\text {offset }}$ to the theoretical Fresnel reflectivity from the $\mathrm{K}$ liquid-vapor interface is shown in Fig. 6. As expected, there is a Debye-Waller-type effect that causes the data to fall below unity with increasing $q_{z}$. However, the measured curve (points) decreases slower than the theoretical prediction (dashed line) for the Debye-Waller-like factor in Eq. (1) convolved with the resolution function.

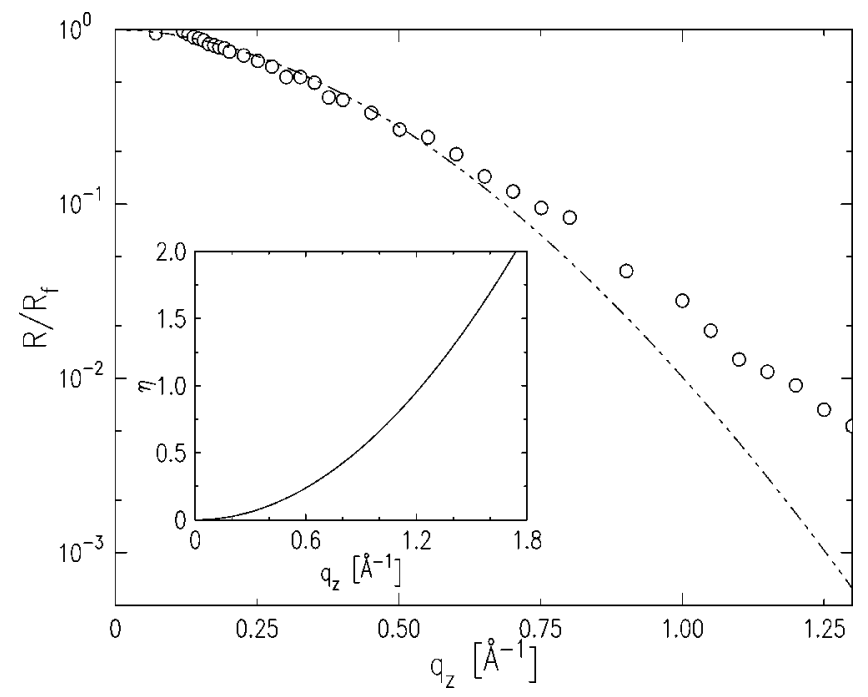

FIG. 6. Fresnel-normalized reflectivity signal $R / R_{f}\left(q_{z}\right)$ for liquid $\mathrm{K}$ (circles) compared to capillary wave predictions (dashed line). The inset shows the capillary wave factor $\eta$ for liquid $\mathrm{K}$ as a function of $q_{z}$.

Figure 7 shows the form factor $\Phi\left(q_{z}\right)$, obtained by dividing the data in Fig. 6 by the integral of the Debye-Waller factor [see Eq. (1)] over the resolution function. In order to compare it with the surface structure factors measured previously for other liquid metals, we have normalized $q_{z}$ by the value of $q_{\text {peak }}$ - the value of $q_{z}$ at which the layering peak is observed or is expected to be observed. In fact we are comparing the electron density structure factors of the different metals. Alternatively we might have compared the atomic densities; however, none of the atomic form factors vary by

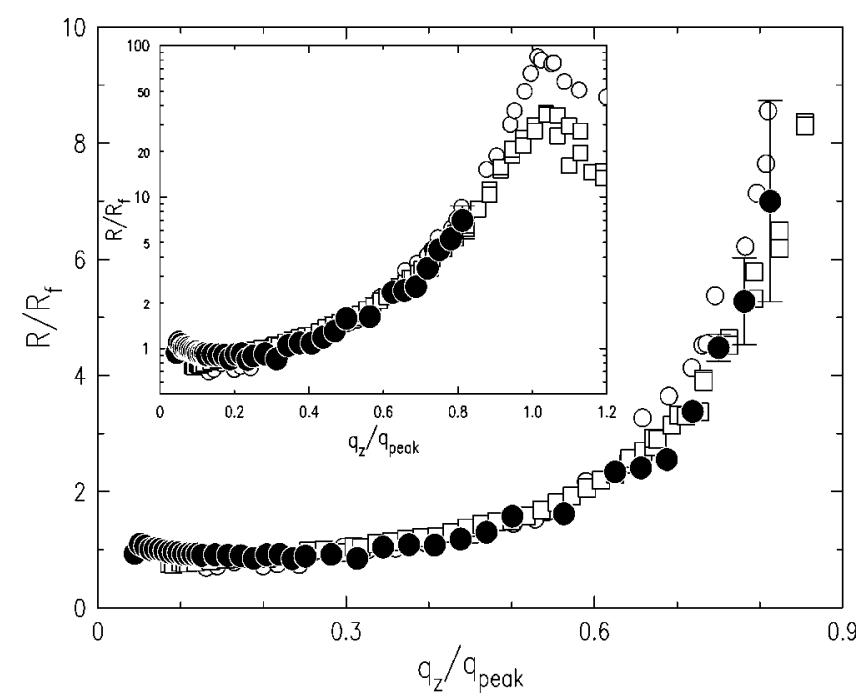

FIG. 7. Surface structure factor $\Phi\left(q_{z}\right)$ for liquid Ga (open circles), liquid In (open squares), and liquid K (filled circles) obtained from $\mathrm{x}$-ray reflectivity data by deconvolving resolution, Fresnel reflectivity, and capillary wave contributions, shown here as a function of $q_{z} / q_{\text {peak }}$; here $q_{\text {peak }}$ is the value of $q_{z}$ at which the layering peak is observed $\left(2.4 \AA^{-1}\right.$ for Ga and $2.2 \AA^{-1}$ for In) or is expected to be observed $\left(1.6 \AA^{-1}\right.$ for $\left.\mathrm{K}\right)$. The inset shows the same three sets of data extended to a greater range. 
more than a few percent over the measured range of $q_{z}$. The surface structure factor of the pure $\mathrm{K}$ is found here to rise clearly above unity and shows a similar functional behavior to that of the pure Ga and In, metals for which the surfaceinduced layering has been well documented. ${ }^{8,9}$ The inset shows the same plot comparing the surface structure factors extracted from the specular reflectivity measurements of pure $\mathrm{Ga}$, pure $\mathrm{In}$, and pure $\mathrm{K}$ over an extended $q_{z} / q_{\text {peak }}$ range. Even though experimental and intrinsic limitations (such as the " $\eta$ effect" discussed above) prevents us from resolving the layering peak in its entirety, it seems clear that liquid $\mathrm{K}$ exhibits surface layering similar to that observed in other metals. These experimental results are supported by computer simulations predicting a surface-induced layering in pure potassium. ${ }^{30}$

\section{DISCUSSION}

In this paper we have expressed the x-ray scattering from the liquid surface, both specular and diffuse, in terms of the product of a thermal fluctuation term and a surface structure term [Eq. (3)]. This is analogous to the usual treatment of Bragg scattering from three-dimensional crystals for which the intensity is expressed as a product of a structure factor and a phonon-induced Debye-Waller factor. For the liquid surface we have assumed that the thermal fluctuations could be described by capillary waves over all length scales extending from a distance $\xi \approx \pi / q_{\max }$ that is of the order of the atomic or molecular size, to a macroscopic distance that is determined by the reciprocal of the reflectometer resolution. The gravitationally determined capillary length is ignored since it is many orders of magnitude longer than the resolution-determined length. In fact the capillary wave model is strictly true only for long wavelengths and the assumption that it can be invoked for lengths as small as $\xi$ is only justified empirically. ${ }^{7,8,10,11,19}$ However, the good agreement between the measured diffuse scattering and that predicted by Eq. (1), found for a number of liquids including the present one provides a rather general justification for adopting this limit. ${ }^{31-34}$

In the present study we generalized the methods that were previously used for liquid surfaces by simultaneously carrying out detailed measurements along and transverse to the specular axis. As in these earlier studies we have been able to verify the $1 / q_{x y}^{2-\eta}$ behavior of the capillary wave form of the surface fluctuations to values of $\eta=0.82$ and to obtain a measure of the surface structure factor. Aside from recent work by Mitrinovic, Williams, and Schlossman ${ }^{29}$ which were carried out to $\eta=0.62$, the largest values of $\eta$ attained in any of the previous studies on liquids were less than half of our value. In view of the fact that the measured value of $\Phi\left(q_{z}\right)$ depends on proper isolation of the capillary diffuse scattering our confirmation of the capillary model for diffuse scattering from $\mathrm{K}$ is essential.

The most significant limitation to the present measurement came about as a result of the fact that due to the thermally excited capillary wave Debye-Waller-like effect, the largest value of $q_{z}$ at which the specular peak can be observed is smaller than $1.6 \AA^{-1}$ at which $\Phi\left(q_{z}\right)$ is expected to have a peak. This limitation can be expressed in terms of the capillary model of Eq. (1) since as $\eta \rightarrow 2$ the specular peak no longer exists. Another way to see the same thing is that the scattering at the peak of $\Phi\left(q_{z}\right)$ will be difficult to observe when the product $\sigma^{2}\left(q_{\text {peak }}\right) q_{\text {peak }}^{2} \gg 1$. In essence, this condition occurs when the effective surface roughness approaches that of the surface layer spacing. Although the precise value of $\sigma\left(q_{\text {peak }}\right)$ depends on the resolution, for the resolutions used to study $\mathrm{K}, \sigma^{2}\left(q_{\text {peak }}\right) q_{\text {peak }}^{2}=13.8$. Nevertheless, in this paper we demonstrated by careful measurement of the diffuse scattering with a number of different resolution configurations that it was possible to quantitatively extract values of $\Phi\left(q_{z}\right)$ for $\mathrm{K}$ over a significant range of $q_{z}$. These measurements establish that over the measured range the $q_{z}$-normalized surface structure factor for pure liquid $\mathrm{K}$ is nearly identical to that found in Ga and In, with a well defined rise above unity as the peak in $q_{z}$ is approached from below. This strongly suggests that the layering in $\mathrm{K}$ is identical to that of other liquid metals, as implied by Velasco et al. ${ }^{5}$ Finally, we note that the present method could also be applied to nonmetallic liquids having similar surface tensions. ${ }^{35-37}$ The unresolved and intriguing question of whether the local surface layering in metallic systems is different from that of nonmetallic liquids when the surface tensions involved are similar will have to await future studies.

\section{ACKNOWLEDGMENTS}

This work has been supported by the U.S. Department of Energy Grant No. DE-FG02-88-ER45379, the National Science Foundation Grant No. DMR-01-12494, and the U.S.Israel Binational Science Foundation, Jerusalem. Work at the CMC Beamlines is supported in part by the Office of Basic Energy Sciences of the U.S. Dept. of Energy and by the National Science Foundation Division of Materials Research. Use of the Advanced Photon Source is supported by the Office of Basic Energy Sciences of the U.S. Department of Energy under Contract No. W-31-109-Eng-38. P.H. acknowledges support from the Deutsche Forschungsgemeinschaft. The help of Arthur Kahaian and Christopher Johnson (CMT Division, Argonne National Laboratory) in preparing the liquid alkali-metal sample, as well as beamline assistance from Thomas Gog and Chitra Venkataraman (CMC-CAT sector, Argonne National Laboratory), are greatly appreciated.

\footnotetext{
${ }^{1}$ S. A. Rice, D. Guidotti, H. Lemberg, W. C. Murphy, and A. N. Bloch, Adv. Chem. Phys. 27, 543 (1974).

${ }^{2}$ R. Evans and M. Hasegawa, J. Phys. C 14, 5225 (1981).

${ }^{3}$ N. H. March, Liquid Metals (Cambridge University Press,
}

Cambridge, 1990).

${ }^{4}$ M. Iwamatsu and S. K. Lai, J. Phys.: Condens. Matter 4, 6039 (1992).

${ }^{5}$ E. Velasco, P. Tarazona, M. Reinaldo-Falagan, and E. Chacon, J. 
Chem. Phys 117, 10777 (2002).

${ }^{6}$ J. G. Harris, J. Gryko, and S. A. Rice, J. Chem. Phys. 87, 3069 (1987).

${ }^{7}$ O. M. Magnussen, B. M. Ocko, M. J. Regan, K. Penanen, P. S. Pershan, and M. Deutsch, Phys. Rev. Lett. 74, 4444 (1995).

${ }^{8}$ M. J. Regan, E. H. Kawamoto, S. Lee, P. S. Pershan, N. Maskil, M. Deutsch, O. M. Magnussen, B. M. Ocko, and L. E. Berman, Phys. Rev. Lett. 75, 2498 (1995).

${ }^{9}$ H. Tostmann, E. DiMasi, P. S. Pershan, B. M. Ocko, O. G. Shpyrko, and M. Deutsch, Phys. Rev. B 59, 783 (1999).

${ }^{10}$ H. Tostmann, E. DiMasi, O. G. Shpyrko, P. S. Pershan, B. M. Ocko, and M. Deutsch, Ber. Bunsenges. Phys. Chem. 102, 1136 (1998).

${ }^{11}$ E. Dimasi, H. Tostmann, O. G. Shpyrko, M. Deutsch, P. S. Pershan, and B. M. Ocko, J. Phys.: Condens. Matter 12, 209 (2000).

${ }^{12}$ N. W. Ashcroft and N. D. Mermin, Solid State Physics (Saunders College Publishing, Orlando, 1976).

${ }^{13}$ M. Hasegawa and M. Watabe, J. Phys. C 15, 353 (1982).

${ }^{14}$ E. Chacon, F. Flores, and G. Navascues, J. Phys. F: Met. Phys. 14, 1587 (1984).

${ }^{15}$ M. A. Gomez and E. Chacon, Phys. Rev. B 49, 11405 (1994).

${ }^{16}$ J. Goodisman and M. L. Rosinberg, J. Phys. C 16, 1143 (1983).

${ }^{17}$ T. Iida and R. I. L. Guthrie, The Physical Properties of Liquid Metals (Oxford University Press, New York, 1993).

${ }^{18} \mathrm{H}$. U. Borgstedt and C. K. Mathews, Applied Chemistry of the Alkali Metals (Plenum, New York, 1987).

${ }^{19}$ H. Tostmann, E. DiMasi, O. G. Shpyrko, P. S. Pershan, B. M. Ocko, and M. Deutch, Phys. Rev. B 61, 7284 (1999).

${ }^{20}$ C. C. Addison, The Chemistry of the Liquid Alkali Metals (John Wiley and Sons, New York, 1984).

${ }^{21}$ P. S. Pershan, Colloids Surf., A 171, 149 (2000).

${ }^{22} \mathrm{~J}$. Als-Nielsen and D. McMorrow, Elements of Modern X-ray
Physics (John Wiley and Sons, Inc., New York, 2001).

${ }^{23}$ P. C. Martin, O. Parodi, and P. S. Pershan, Phys. Rev. A 6, 2401 (1972).

${ }^{24}$ M. Fukuto, R. K. Heilmann, P. S. Pershan, J. A. Griffiths, S. M. Yu, and D. A. Tirrell, Phys. Rev. Lett. 81, 3455 (1998).

${ }^{25}$ S. K. Sinha, E. B. Sirota, S. Garoff, and H. B. Stanley, Phys. Rev. B 38, 2297 (1988).

${ }^{26}$ J. Als-Nielsen, F. Christensen, and P. S. Pershan, Phys. Rev. Lett. 48, 1107 (1982).

${ }^{27}$ D. K. Schwartz, M. L. Schlossman, E. H. Kawamoto et al., Phys. Rev. A 41, 5687 (1990).

${ }^{28}$ D. Forster, Hydrodynamic Fluctuations, Broken Symmetry, and Correlation Functions (Imprint, W. A. Benjamin, Reading, MA, Advanced Book Program, 1975).

${ }^{29}$ D. M. Mitrinovic, S. M. Williams, and M. L. Schlossman, Phys. Rev. E 6302, 1601 (2001).

${ }^{30}$ D. Chekmarev, M. Zhao, and S. Rice, J. Chem. Phys. 109, 768 (1998).

${ }^{31}$ M. K. Sanyal, S. K. Sinha, K. G. Huang, and B. M. Ocko, Phys. Rev. Lett. 66, 628 (1991).

${ }^{32}$ B. M. Ocko, X. Z. Wu, E. B. Sirota, S. K. Sinha, and M. Deutsch, Phys. Rev. Lett. 72, 242 (1994).

${ }^{33}$ A. K. Doerr, M. Tolan, W. Prange, J. P. Schlomka, T. Seydel, W. Press, D. Smilgies, and B. Struth, Phys. Rev. Lett. 3, 3470 (1999).

${ }^{34}$ B. R. McClain, M. Yoon, J. D. Litster, and S. G. J. Mochrie, Eur. Phys. J. B 10, 45 (1999).

${ }^{35}$ E. Chacon, M. Reinaldo-Falagan, E. Velasco, and P. Tarazona, Phys. Rev. Lett. 87, 166101 (2001).

${ }^{36}$ J. M. Soler, G. Fabricius, and E. Artacho, Surf. Sci. 482, 1314 (2001).

${ }^{37}$ G. A. Chapela, G. Saville, S. M. Thompson, and J. S. Rowlinson, J. Chem. Soc., Faraday Trans. 2 73, 1133 (1977). 